\title{
Diffusional Kurtosis Imaging of the Developing Brain
}

\author{
A. Paydar, E. Fieremans, J.I. Nwankwo, M. Lazar, H.D. Sheth, V. Adisetiyo, J.A. Helpern, J.H. Jensen, and S.S. Milla
}

\begin{abstract}
BACKGROUND AND PURPOSE: Diffusional kurtosis imaging is an extension of DTI but includes non-Gaussian diffusion effects, allowing more comprehensive characterization of microstructural changes during brain development. Our purpose was to use diffusional kurtosis imaging to measure age-related microstructural changes in both the WM and GM of the developing human brain.
\end{abstract}

MATERIALS AND METHODS: Diffusional kurtosis imaging was performed in 59 subjects ranging from birth to 4 years 7 months of age. Diffusion metrics, fractional anisotropy, and mean kurtosis were collected from VOls within multiple WM and GM structures and subsequently analyzed with respect to age. Diffusional kurtosis tractography images at various stages of development were also generated.

RESULTS: Fractional anisotropy and mean kurtosis both showed age-related increases in all WM regions, reflecting progression of diffusional anisotropy throughout development, predominantly in the first 2 years of life (eg, $70 \%$ and $157 \%$ increase in fractional anisotropy and mean kurtosis, respectively, from birth to 2 years for the splenium). However, mean kurtosis detected continued microstructural changes in WM past the fractional anisotropy plateau, accounting for more delayed isotropic changes (eg, $90 \%$ of maximum fractional anisotropy was reached at 5 months, whereas $90 \%$ of maximum mean kurtosis occurred at 18 months for the external capsule). Mean kurtosis may also provide greater characterization of GM maturation (eg, the putamen showed no change in fractional anisotropy but an $81 \%$ change in mean kurtosis from birth to 4 years 7 months).

CONCLUSIONS: Mean kurtosis detects significant microstructural changes consistent with known patterns of brain maturation. In comparison with fractional anisotropy, mean kurtosis may offer a more comprehensive evaluation of age-related microstructural changes in both WM and GM and is potentially a valuable technique for studying brain development.

ABBREVIATIONS: DKI = diffusional kurtosis imaging; FA = fractional anisotropy; IC = internal capsule; max = maximum; MK = mean kurtosis

$\mathbf{M}_{\mathrm{s}}^{\mathrm{R}}$ $\mathrm{R}$ imaging has become the criterion standard for noninvasive high-resolution brain imaging in the pediatric population. Macrostructural changes that take place within the brain during maturation have been well documented by conventional

Received June 29, 2013; accepted after revision August 14.

From the Department of Radiology (A.P., E.F., J.I.N., M.L., H.D.S., V.A., S.S.M.), Center for Biomedical Imaging, New York University School of Medicine, New York, New York; and Department of Radiology and Radiological Science (J.A.H., J.H.J.), Center for Biomedical Imaging, Medical University of South Carolina, Charleston, South Carolina.

The authors do not have a direct financial/commercial interest or financial involvement with any organization.

Preliminary data from this research previously presented at: Annual Meeting of the American Society of Neuroradiology and the Foundation of the ASNR Symposium, April 16-21, 2012; New York, New York.

Please address correspondence to Amir Paydar, MD, Department of RadiologyNeuroradiology Section, Center for Biomedical Imaging, NYU Langone Medical Center, 660 First Ave, 3rd Fl, New York, NY 10016; e-mail: amir.paydar@nyumc.org http://dx.doi.org/10.3174/ajnr.A3764
MR imaging techniques in both normal and pathologic states. ${ }^{1-6}$ However, these conventional techniques are limited in their ability to quantify developmental changes that occur at the microstructural level. Therefore, in vivo characterization and accurate diagnosis of microstructural abnormalities currently remain challenging.

During brain development, many cellular processes can affect water diffusion properties. WM myelination and maturation, axonal growth and development, and changes in axonal membrane permeability can affect free water diffusion. ${ }^{7}$ Indeed, DWI has been used to detect many of these microstructural changes that occur during brain maturation. ${ }^{8}$ In particular, the widely used DTI technique has been shown to be sensitive to age-related microstructural changes in both rodent and human models. ${ }^{5,7,9-11}$

DTI describes restricted diffusion in the brain by estimating the diffusion tensor, whereby the principal diffusion tensor eigenvector tends to be directed parallel to axonal bundles (axial direc- 
tion) and the other eigenvectors are perpendicular to the axons (radial direction). ${ }^{12}$ The diffusivities corresponding to these directions are referred to as axial diffusivity and radial diffusivity, respectively. ${ }^{12,13}$ It has been proposed that axial diffusivity characterizes axonal architecture, while radial diffusivity reflects myelin integrity, $9,12,14$ though such simplified interpretations should be regarded with caution. ${ }^{15}$ From diffusion tensor eigenvalues, fractional anisotropy (FA), which is a primary index of diffusional directionality, may be calculated. FA values range from zero to 1 , where zero implies an isotropic environment with similar diffusion in all directions and 1 implies diffusion along only 1 axis. ${ }^{13,16}$ By using these diffusion tensor eigenvectors, one can evaluate the anisotropic neuroarchitectural orientation of WM fiber tracts. Accordingly, DTI is an excellent tool for investigating the agerelated increase in anisotropy that occurs within the network of WM tracks as a result of myelination. ${ }^{5,9,17}$ However, DTI is based on a Gaussian approximation of water diffusion, which limits its sensitivity to diffusional and microstructural properties of biologic tissues. ${ }^{18}$

Recently, diffusion-weighted techniques that exploit diffusional non-Gaussianity have been developed, including high-angular-resolution diffusion imaging, diffusion spectrum imaging, and diffusional kurtosis imaging (DKI). ${ }^{19-22}$ These techniques take into account the non-Gaussian diffusional properties of water motion in complex media and are, therefore, more comprehensive in evaluating brain tissue microstructural complexity..$^{9,19,23}$ The DKI technique is a clinically feasible extension of the traditional DTI model while maintaining the ability to estimate all the standard diffusion tensor metrics, including axial diffusivity, radial diffusivity, and FA, ${ }^{19,20,24}$ but with improved accuracy. ${ }^{18}$ DKI requires at least 2 nonzero b-values and 15 diffusion gradient directions (compared with 1 nonzero b-value and 6 directions for DTI), thereby affording a higher angle resolution of diffusion acquisition. ${ }^{18,19,23}$ Moreover, DKI provides an additional metric that quantifies non-Gaussian diffusion termed diffusional kurtosis, $K$. This metric is defined by the equation

$$
K=\frac{M_{4}}{M_{2}^{2}}-3
$$

where $K$ represents excess kurtosis and $M_{n}$ is the nth-order diffusion displacement moment; in a purely Gaussian distribution, $K=0 .{ }^{19}$ Accordingly, $K$ is a measure that quantifies the deviation of the water diffusion profile from a Gaussian distribution, potentially allowing sensitivity to underlying microstructural barriers not detected by the standard diffusion tensor metrics. By using this parameter, one can generate the metrics axial kurtosis, radial kurtosis, and mean kurtosis (MK), which are the kurtosis counterparts of axial diffusivity, radial diffusivity, and FA, respectively.

In this human study, we used DKI to quantify microstructural changes that occur in both WM and GM during the first 5 years of life, when the brain is in its most active stage of development. We hypothesize that owing to their sensitivity for the detection of microstructural changes in both anisotropic and isotropic environments, ${ }^{10,19}$ the kurtosis metrics of DKI (particularly MK) may provide additional information about brain maturation compared with that obtainable with conventional diffusion tensor metrics (specifically FA). Therefore, DKI has the potential to im- prove the evaluation of the developing brain in both normal and pathologic states.

\section{MATERIALS AND METHODS}

An institutional review board-approved retrospective review was initiated by obtaining a comprehensive data base of brain MRI in 59 pediatric subjects ( 31 female, 28 male) who underwent DKI imaging as part of a routine MR imaging examination under sedation at a major university medical center from June 2009 to October 2010. Subject or parental informed consent was waived because it was not required by the institutional review board. The age range of our subjects was birth (ie, day 1 of life) to 4 years 7 months (ie, 1689 days of age). Subjects who underwent brain MR imaging for non-neurologic indications (eg, facial hemangiomas, orbital lesions, sinonasal abnormalities, vomiting, weight loss, precocious puberty) were included in this study. However, premature infants and subjects who had medical histories with possibly related intracranial/neurologic manifestations (eg, seizures or delayed myelination) were excluded from this study. All the included examination findings were interpreted as normal by fellowship-trained board-certified neuroradiologists. MR imaging examinations that demonstrated any intracranial pathology or were considered of low quality due to motion artifacts, oblique positioning, or a low signal-to-noise ratio were also excluded. All MR imaging examination findings were re-evaluated by a board-certified pediatric neuroradiologist for normalcy before inclusion.

All studies were performed on a 1.5T MR imaging scanner (Magnetom Avanto; Siemens, Erlangen, Germany). A body coil was used for transmission of the signal and an 8-channel head coil was used to receive the signal. Diffusion-weighted data were obtained using an axial fat-suppressed single-shot echo-planar sequence with the following imaging parameters: TR/TE $=4500 / 96$ ms; matrix size $=78-82 \times 78-82 \times 28-34$; voxel size $=2.2-$ $2.8 \times 2.2-2.8 \times 4-5 \mathrm{~mm}^{3}$ (ie, section thickness $=4-5 \mathrm{~mm}$, without an intersection gap); generalized autocalibrating partially parallel acquisitions acceleration factor $=2$; acquisition time $=4$ minutes 48 seconds; diffusion directions $=30$; b-values $=0$, 1000 , and $2000 \mathrm{~s} / \mathrm{mm}^{2}$. Of note, maximum b-values of approximately $2000 \mathrm{~s} / \mathrm{mm}^{2}$ are needed for the diffusion signal to be sensitive to non-Gaussian effects and to quantify accurate kurtosis values. ${ }^{19,23}$

DKI data were processed by using in-house software called the Diffusional Kurtosis Estimator (http://www.nitrc.org/projects/ dke). Subsequently, the diffusion and kurtosis tensors were calculated on a voxel-by-voxel basis to produce skull-stripped parametric gray-scale maps for FA and MK. ${ }^{19,25}$ Sample transaxial sections from FA and MK maps for the youngest and oldest subjects are portrayed in Fig $1 A$ and $-B$.

Using MRIcron (http://www.sph.sc.edu/comd/rorden/mricro. html), we drew VOIs directly on multiple FA map transverse sections for volumetric analysis. VOIs were drawn on 9 different anatomic WM and GM structures, including the genu and splenium of the corpus callosum, frontal WM, parietal WM, anterior and poster limbs of the internal capsule (IC), external capsule, thalamus, and putamen (Fig 1C). VOI drawing was performed by a neuroradiology fellow, fourth-year radiology res- 


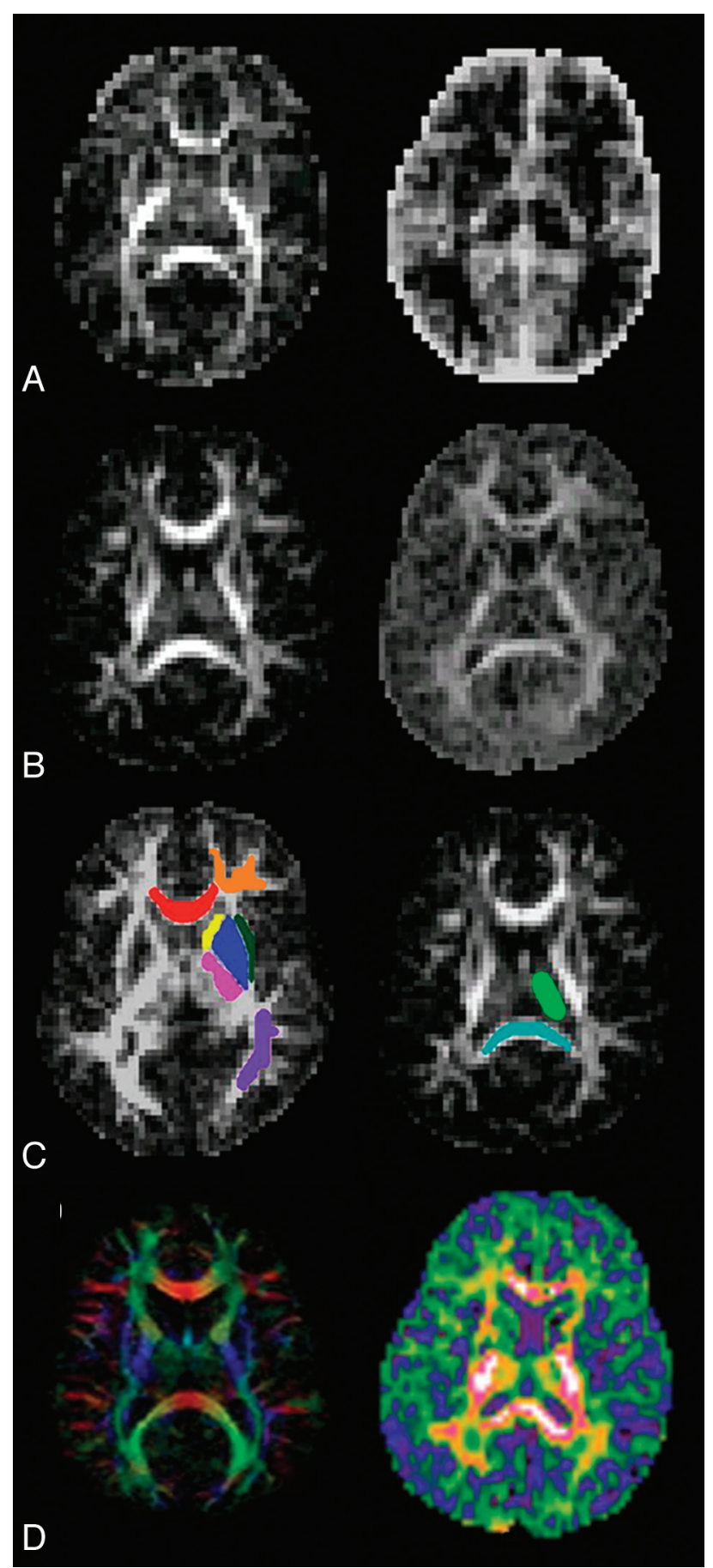

FIG 1. A, Transaxial FA (left) and MK (right) maps at birth. $B$, Transaxial FA (left) and MK (right) maps at 4 years 7 months. C, Examples of VOIs drawn on transaxial FA maps over the genu (red), frontal WM (orange), parietal WM (purple), putamen (blue), anterior IC (yellow), posterior IC (pink), external capsule (dark green), thalamus (light green), and splenium (cyan). D, Transaxial FA (left) and MK (right) color maps.

ident, and a second-year medical student. To control for interobserver variability, we assigned each of these investigators to draw all of the VOIs for any given anatomic structure for all the subjects. All VOIs were later carefully reviewed by a single boardcertified pediatric neuroradiologist to further minimize interobserver variability. VOIs were then applied to the parametric maps in Matlab (MathWorks, Natick, Massachusetts) to calculate the mean FA and MK values for each VOI.
Thereafter, the mean FA and MK for each VOI were analyzed for each subject and correlated with age by fitting each parameter value $P$ to a nonlinear monoexponential plus a constant, $P=$ $a e^{-b X}+c$, where $X$ is age and $a, b, c$ are fitting parameters. Using this function, we generated regression curves for both FA and MK values for all 9 VOIs in Matlab (Figs 2-5). Coefficient of determination $\left(R^{2}\right)$ values were also calculated to demonstrate the quality of the exponential fit for the FA and MK regression curves (Table). In addition, to compare developmental timing parameters for all $9 \mathrm{WM}$ and GM VOIs, we first defined the developmental plateau for both FA and MK values at the asymptotes of the exponential curves and labeled them as $\mathrm{FA}_{\max }$ and $\mathrm{MK}_{\max }$, respectively (Table). Using these $\mathrm{FA}_{\max }$ and $\mathrm{MK}_{\max }$ values, we calculated percentage changes in both FA and MK metrics at 2 years and 4 years 7 months of age. Finally, the specific ages at which $90 \%$ of $\mathrm{FA}_{\max }$ and $\mathrm{MK}_{\max }$ were reached since birth were estimated for all VOIs.

DKI tractography was performed for the newborn, 6 month, 11 month, and 2 year 1 month subjects (Fig 6). Complex orientation distribution function profiles were obtained at all brain voxels with MK $>0.1,{ }^{26}$ and fiber directions at each voxel were estimated by using the maxima of the orientation distribution function profiles. VOIs for the genu, splenium, anterior IC, posterior IC, and external capsule were used as seeds to generate fiber trajectories by using a fiber assignment by continuous tracking approach. ${ }^{27}$ Fiber tracts were terminated if they reached regions with $\mathrm{FA}<0.1$ (for subjects at least several months of age) or FA $<0.05$ (for the neonate subject) or if the angle between 2 consecutive steps was $>45^{\circ}$. Only tracts with lengths larger than $30 \mathrm{~mm}$ were retained in the final image.

\section{RESULTS}

Figures $2-5$ show the data points for the 59 subjects with the corresponding regression curves, demonstrating the progressive changes in the FA and MK values for all $9 \mathrm{WM}$ and GM regions as a function of age from birth to 4 years 7 months. Figure 2 shows the FA curves as manifested by a change in the FA value with time in all $7 \mathrm{WM}$ regions. There is an exponential increase in FA during the course of development within all WM structures, most appreciable within the first 2 years. Figure 3 shows the MK curves, demonstrating a change in the MK value with time in the same 7 WM regions. There is also a steady increase in MK with time within all WM structures, also most evident in the first 2 years.

The corpus callosum demonstrates relatively higher FA and MK values compared with the other WM regions, the splenium more so than the genu. Differences in the sloping of both FA and MK curves are also seen between the splenium and genu. The splenium shows an earlier percentage rise in both FA and MK compared with the genu. In addition, $90 \%$ of the developmental plateaus for both FA and MK curves (ie, $90 \%$ of $\mathrm{FA}_{\max }$ and $\left.\mathrm{MK}_{\max }\right)$ are reached at an earlier age for the splenium (9 and 15 months) than for the genu (16 and 19 months; Table).

Important temporal differences are also observed between FA and MK curves. As demonstrated in the Table, MK developmental plateaus are reached at later ages than those for FA in all WM regions. Percentage change in FA reaches its asymptote at approximately 2 years of age, while percentage change in MK continues beyond the 2-year mark. In addition, in all WM 


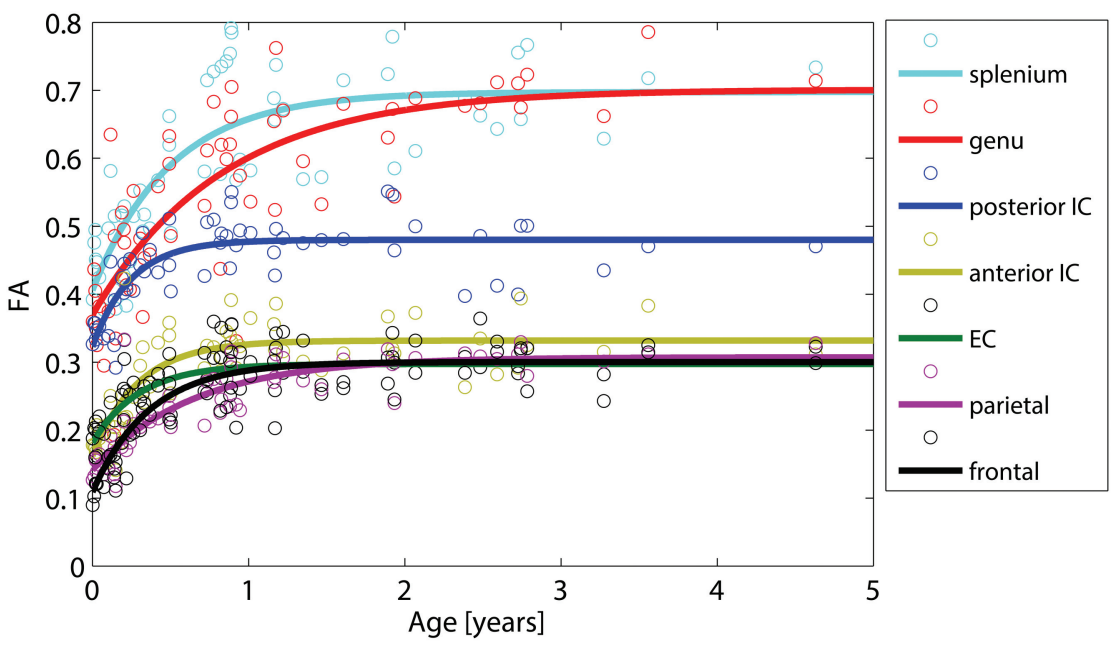

FIG 2. FA curves demonstrate a change in FA value as a function of age in all 7 WM regions.

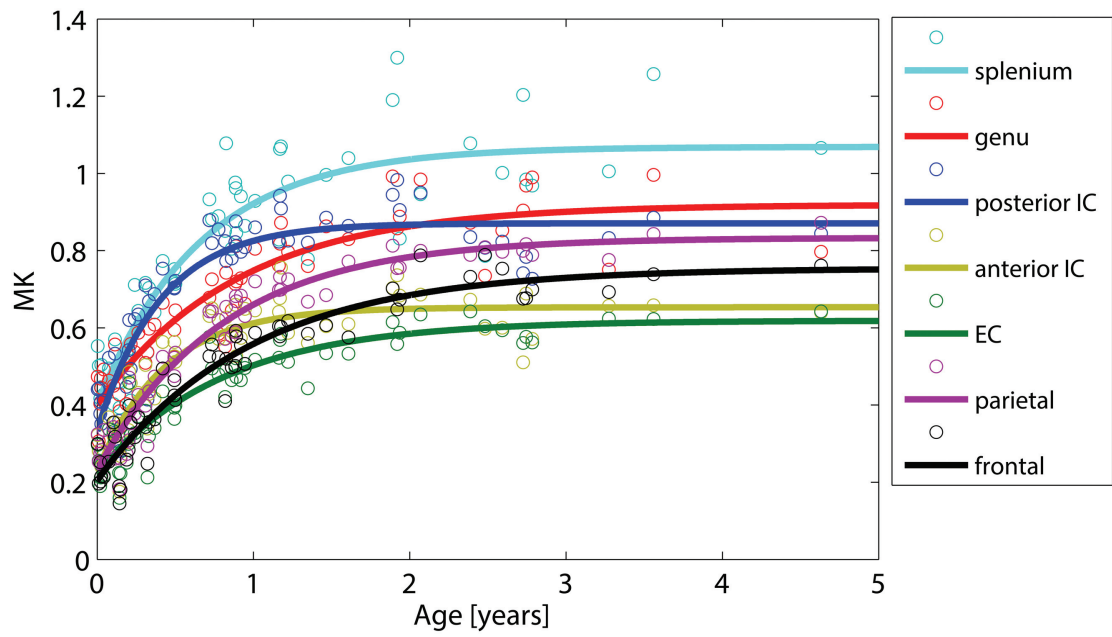

FIG 3. MK curves demonstrate a change in MK value as a function of age in all 7 WM regions.

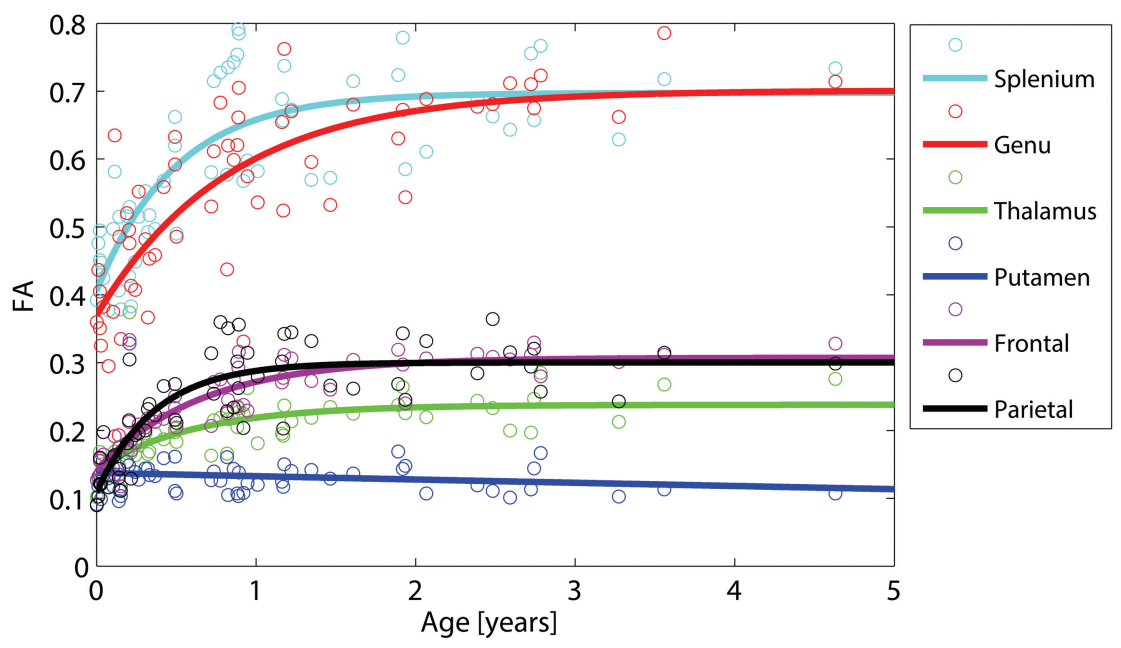

FIG 4. FA curves demonstrate a change in FA values as a function of age in $2 \mathrm{GM}$ regions (thalamus and putamen); FA curves for 4 WM regions from Fig 3 are also included in this graph for comparison.

locations, $90 \%$ of $\mathrm{MK}_{\max }$ is reached at a later age (eg, at 18 months in the external capsule) than $90 \%$ of $\mathrm{FA}_{\max }$ (eg, 5 months in the external capsule).
Figures 4 and 5 show the FA and MK curves for the 2 GM regions, the thalamus and putamen; FA and MK curves for the subcortical WM regions and the corpus callosum are also included in these graphs for comparison. No detectible change in FA is seen in the putamen with time. In contrast, there is up to an $81 \%$ change in MK detected within the putamen from birth to 4 years 7 months (Table). Notably, the FA curve for the thalamus demonstrates a slight percentage increase in FA (63\% at 2 years and $66 \%$ at 4 years 7 months). On the other hand, the MK curve for the thalamus shows a much more substantial increase in MK $(136 \%$ at 2 years and $137 \%$ at 4 years 7 months).

DKI tractography images in Fig 6 qualitatively illustrate the progressive increase in volume and coherent orientation of WM fibers within the genu, splenium, anterior IC, posterior IC, and external capsule WM structures from birth to 2 years 1 month of age.

\section{DISCUSSION}

With respect to conventional DTI, DKI has been shown to provide additional information about microstructural changes in the developing brain. A previous rodent study ascertained that DKI offers a more sensitive evaluation of the microstructural complexity of both WM and GM at 3 stages of brain development compared with DTI. ${ }^{10}$ We were able to document similar findings in the human brain.

In our study, a progressive rise in FA throughout all WM regions reflects the increase in anisotropy in WM tracts as myelination progresses. This phenomenon has also been well-documented in prior investigations. ${ }^{10,11}$ Notably, this trend is most evident in the first 2 years, when myelination is the dominant contributor to the increase in the microstructural complexity of WM. In addition, the relatively higher FA in the corpus callosum throughout all ages may be attributed to its more tightly packed and anisotropic architecture. ${ }^{5,9,10,28}$ We also observed that the increase in FA and the age at which FA peaks both occur relatively earlier in the splenium than in the genu, in keeping with the well-known caudorostral pattern of myelination of the corpus callosum during maturation. ${ }^{1,4,7,11,28,29}$ 


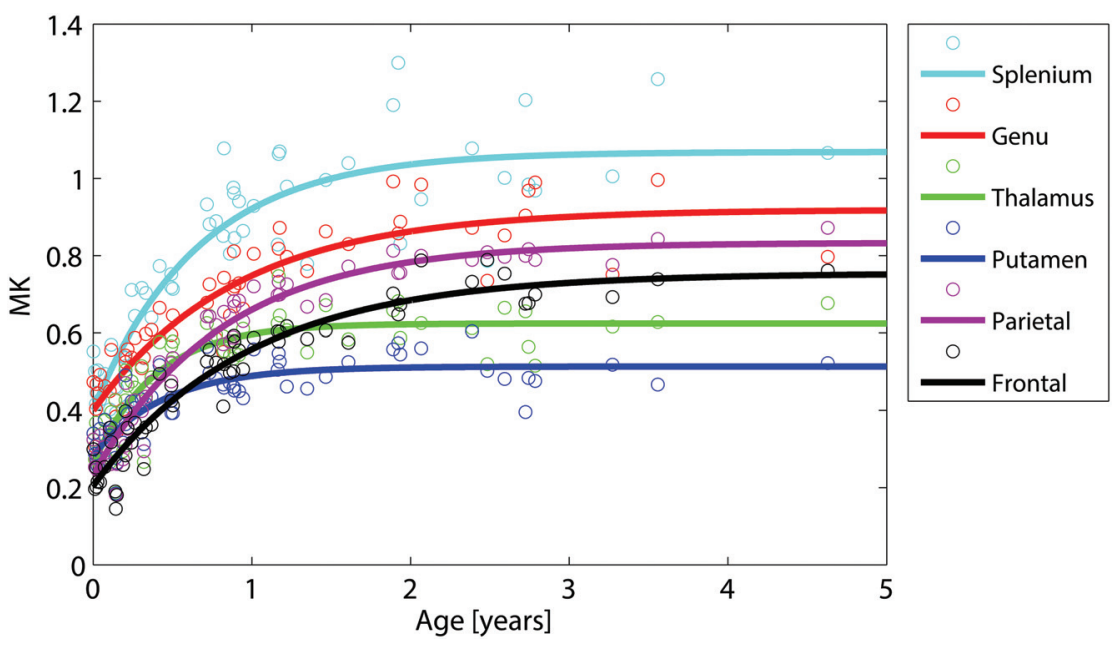

FIG 5. MK curves demonstrate a change in MK values as a function of age in 2 GM regions (thalamus and putamen); MK curves for 4 WM regions from Fig 4 are also included in this graph for comparison.

MK, like FA, increases in all WM regions, suggesting that DKI can detect the age-related increase in anisotropy as well, likely also predominantly as a function of myelination. DKI tractography images (Fig 6) display this age-related increase in anisotropy within central WM tracts, as reflected by the increase in tract coherence with age. However, our DKI data also show that MK continues to rise beyond the 2-year mark and plateaus at later ages than FA does in all WM locations. This trend indicates that DKI can further resolve isotropic diffusion barriers that continue to develop in the WM even after myelination and axonal packing have already peaked. Speculatively, these barriers may form partly as a result of an increase in the complexity of intrinsic cellular processes and extracellular matrices, ${ }^{18-20}$ axonal pruning, ${ }^{10}$ and functional reorganization of myelin to allow the progressive increase in axon conduction velocities, ${ }^{11,30,31}$ all of which continue to occur during later stages of development. Another concept that may explain this trend is the continued maturation of crossing fibers in WM during later childhood. DTI is limited in the evaluation of both anisotropy and directionality of crossing fibers because the diffusion tensor can only resolve a singlefiber orientation within each voxel. ${ }^{10,21,22}$ While FA is diminished inside voxels containing crossing fibers, MK can better define the multidirectional environment inside these voxels. This intrinsic prop- erty is also validated with tractography. Via the application of the orientation distribution function, fiber tracking with DKI has been shown to better account for the presence of these crossing fibers. ${ }^{26}$ DTI and DKI tractography images in Fig 7 visually demonstrate this concept by displaying the higher sensitivity of DKI for crossing-fiber resolution. In conclusion, as isotropic diffusion barriers and more complex fiber patterns continue to materialize in the WM after myelination has already been established, a non-Gaussian diffusion approach may better characterize these more delayed developmental changes.

Our study also supports the hypothesis that DKI is sensitive to age-related microstructural changes that occur in the isotropic GM, for which DTI has previously been shown to have limited sensitivity. ${ }^{9,10}$ In the putamen, there is no appreciable change in FA throughout the first 4 years 7 months of life, corresponding to the complete lack of anisotropic architecture of the putamen. However, a slight increase in FA is seen in the thalamus with time, mainly because the thalamus, though predominantly a GM structure, has a sizeable fraction of linear axonal WM tracts and therefore an element of internal anisotropy. ${ }^{9}$ On the other hand, there is a steady rise in MK in both the putamen and thalamus with time, accounting for other specific isotropic changes that occur in these GM structures throughout development. In theory, these isotropic diffusion barriers to which $\mathrm{MK}$ is sensitive probably emerge as a result of a progressive increase in macromolecular concentration and a decrease in tissue water content as GM matures. ${ }^{9}$ Other specific cytoarchitectural changes that affect MK-sensitive isotropic diffusion behaviors in the developing GM may include the proliferation of cell membranes and organelles, the transition of radial glial cells to astrocytic neuropil, the addition of basal dendrites, and cell packing. ${ }^{9,10,14}$ Therefore, compared with FA, MK can better resolve the progression of GM organization with respect to age by accounting for other isotropic microstructural barriers that form at the cellular level.

Relative change in FA and MK from birth to 4 years 7 months at all anatomic locations

\begin{tabular}{|c|c|c|c|c|c|c|c|c|}
\hline \multirow[b]{2}{*}{ Anatomic Location } & \multicolumn{4}{|c|}{ FA } & \multicolumn{4}{|c|}{ MK } \\
\hline & $R^{2 \mathrm{a}}$ & $\begin{array}{c}\% \Delta \\
\text { (Birth to } 2 \text { yr) })^{b}\end{array}$ & $\begin{array}{c}\% \Delta \\
{\text { (Birth to } 4 \text { yr } 7 \mathrm{mo})^{\mathrm{c}}}\end{array}$ & $\begin{array}{c}90 \% \text { of } \\
\mathrm{FA}_{\max }(\mathrm{mo})^{\mathrm{d}}\end{array}$ & $R^{2 \mathrm{a}}$ & $\begin{array}{c}\% \Delta \\
{\text { (Birth to } 2 \mathrm{yr})^{\mathrm{b}}}\end{array}$ & $\begin{array}{c}\% \Delta \\
\text { (Birth to } 4 \mathrm{yr} 7 \mathrm{mo})^{\mathrm{c}}\end{array}$ & $\begin{array}{c}90 \% \text { of } \\
\mathrm{MK}_{\max }(\mathrm{mo})^{\mathrm{d}}\end{array}$ \\
\hline Splenium & 0.66 & 70 & 71 & 9 & 0.82 & 157 & 165 & 15 \\
\hline Genu & 0.63 & 81 & 89 & 16 & 0.85 & 115 & 129 & 19 \\
\hline Frontal WM & 0.69 & 172 & 173 & 8 & 0.92 & 233 & 265 & 23 \\
\hline Parietal WM & 0.77 & 111 & 116 & 13 & 0.94 & 236 & 257 & 19 \\
\hline Anterior IC & 0.62 & 103 & 103 & 6 & 0.85 & 182 & 184 & 10 \\
\hline Posterior IC & 0.61 & 48 & 48 & 4 & 0.84 & 158 & 159 & 9 \\
\hline EC & 0.59 & 63 & 63 & 5 & 0.87 & 160 & 174 & 18 \\
\hline Thalamus & 0.45 & 63 & 66 & 10 & 0.78 & 136 & 137 & 8 \\
\hline Putamen & 0.03 & -7 & -18 & N/A & 0.68 & 80 & 81 & 8 \\
\hline
\end{tabular}

Note:-NA indicates not applicable; EC external capsule.

${ }^{a}$ Coefficient of determination $\left(R^{2}\right)$ demonstrating the goodness of exponential fit for the FA and MK nonlinear monoexponential regression curves.

b Percentage change in FA and MK absolute values from birth to 2 years of age.

c Percentage change in FA and MK absolute values from birth to 4 years 7 months of age.

${ }^{\mathrm{d}}$ Approximate age in months at which $90 \%$ of developmental plateau is reached since birth for both $\mathrm{FA}$ and $\mathrm{MK}$ datasets (ie, $\mathrm{FA}_{\text {max }}$ and $\mathrm{MK}_{\text {max }}$ ). 


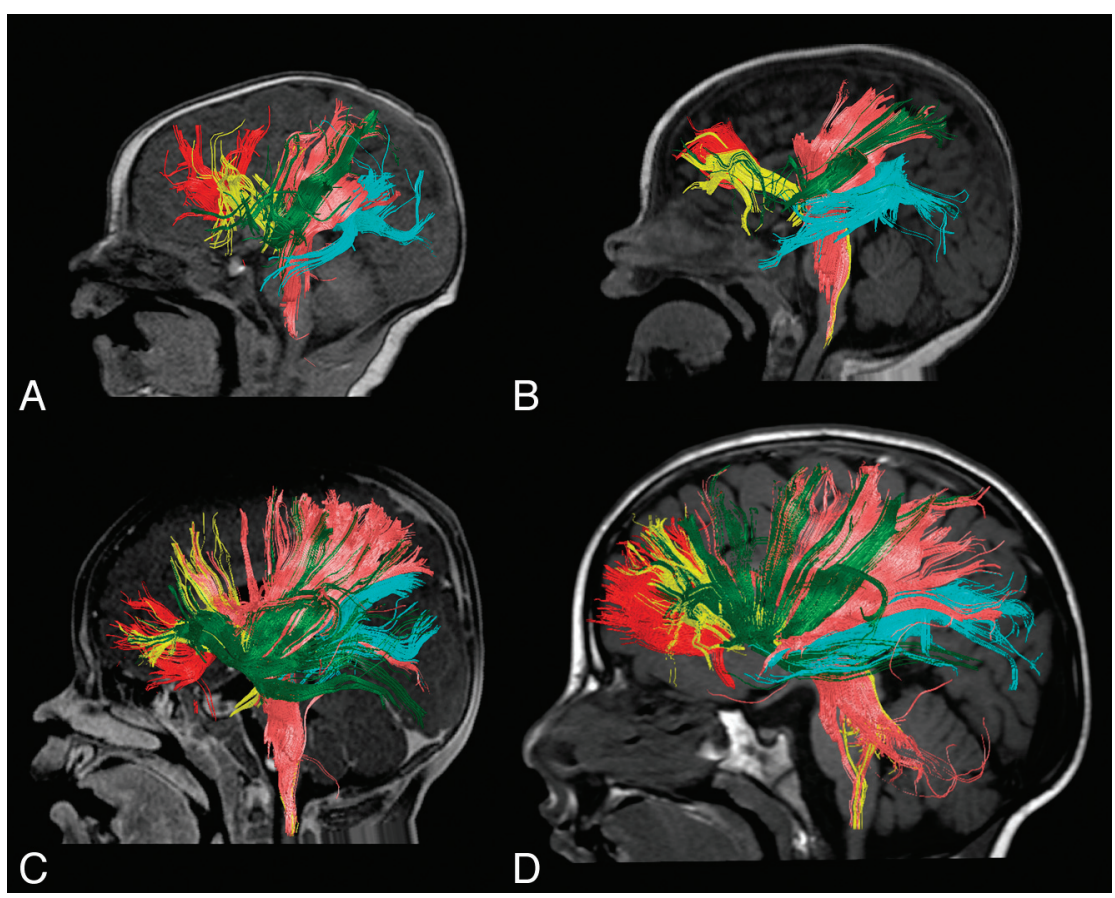

FIG 6. DKI tractography at various stages of development, including birth $(A), 6$ months $(B), 11$ months $(C)$, and 2 years 1 month $(D)$. Fiber tracking is displayed for the genu (red), splenium (cyan), anterior IC (yellow), posterior IC (pink), and external capsule (dark green).
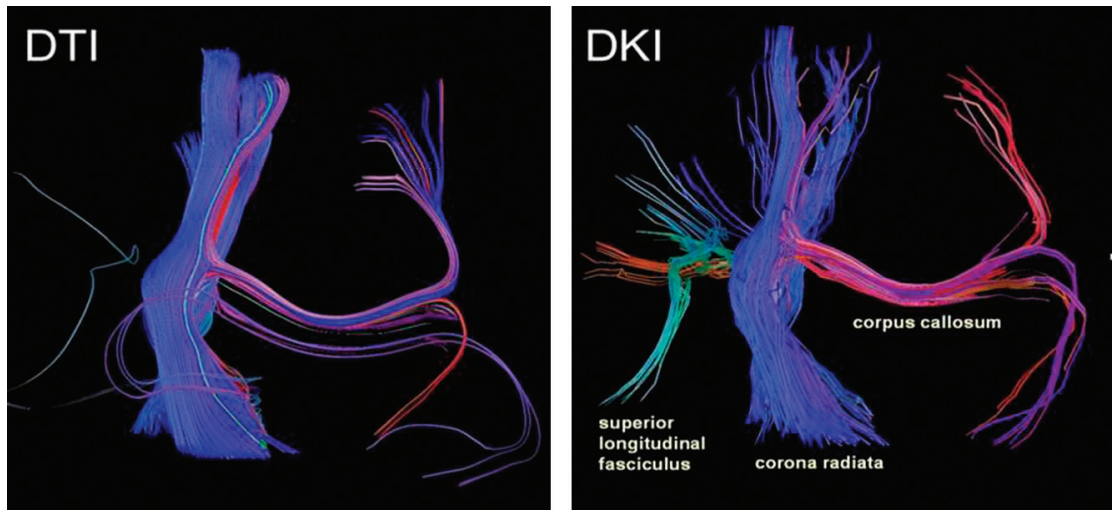

FIG 7. DTI and DKI tractographies performed on a normal brain are provided as an example to illustrate the difference in sensitivity for the detection of tiny crossing fibers between DTI and DKI fiber-tracking techniques.

This investigation was partly limited due to its retrospective nature and lack of longitudinal information, particularly pertaining to the neurologic development of the included subjects. In addition, VOI drawing by 3 independent investigators may have introduced some degree of interobserver variability in our study results, though no definite discrepancy was identified in their drawing techniques. Finally, VOI drawing on FA maps may have been influenced by subjective selection of structures defined by variable FA contrast at different ages.

Future DKI studies should be performed to further expand our understanding of non-Gaussian diffusivity in the developing brain. Previous research has shown that DTI can detect age-related microstructural changes in the brain beyond 5 years of age. In a study by Lebel et al, subtle gradual changes in FA were observed within most WM and GM locations up to early adulthood. Therefore, the use of DKI parameters to study normal developmental changes in the brain of subjects older than 5 years is another subject worth pursuing. In addition, we aim to use DKI for the evaluation of other GM regions in our future investigations. DKI also remains a promising technique for studying the developing brain in pathologic states. ${ }^{32}$ It has already been shown to yield valuable information about the microstructural integrity of the brain in children with epilepsy $^{33}$ and in adolescents with attention deficit/hyperactivity disorder. ${ }^{34}$ Indeed, future DKI studies may explore the sensitivity of kurtosis metrics to pathologies that alter the microstructural complexity in the developing brain.

\section{CONCLUSIONS}

This human study replicates findings made in previous rodent studies, reflecting the additional information that DKI provides for detecting microstructural changes in the developing human brain. Similar to DTI, DKI can also detect anisotropic WM changes due to myelination, predominantly during the first 2 years of life. In addition, DKI can identify other isotropic WM changes that occur beyond the first 2 years. Finally, DKI may also provide greater characterization of GM maturation. In summary, DKI offers sensitive and comprehensive measures for the quantitative evaluation of agerelated microstructural changes in both WM and GM and thereby may be a valuable technique for studying the developing brain.

Disclosures: Els Fieremans-UNRELATED: Patents (planned, pending or issued): Siemens Medical owns a royalty-free nonexclusive license for diffusional kurtosis imaging with the pending patent held by New York University. I am one of the inventors. * Money paid to the institution.

\section{REFERENCES}

1. Brody BA, Kinney HC, Kloman AS, et al. Sequence of central nervous system myelination in infancy. I. An autopsy study of myelination. J Neuropathol Exp Neurol 1987;46:283-301

2. Barkovich AJ, Kjos BO, Jackson DE, et al. Normal maturation of the neonatal and infant brain: MR imaging at 1.5 T. Radiology 1988; 166(1 pt 1):173-80

3. Dobbing J, Sands J. Quantitative growth and development of human brain. Arch Dis Child 1973;48:757-67

4. Kinney HC, Brody BA, Kloman AS, et al. Sequence of central nervous system myelination in human infancy. II. Patterns of myelination in autopsied brains. J Neuropathol Exp Neurol 1988;47:217-34

5. Lebel C, Walker L, Leemans A, et al. Microstructural maturation of the human brain from childhood to adulthood. Neuroimage 2008; 40:1044-55 
6. Deipolyi AR, Mukherjee P, Gill K, et al. Comparing microstructural and macrostructural development of the cerebral cortex in premature newborns: diffusion tensor imaging versus cortical gyration. Neuroimage 2005;27:579-86

7. Takeda K, Nomura Y, Sakuma H, et al. MR assessment of normal brain development in neonates and infants: comparative study of T1- and diffusion-weighted images. J Comput Assist Tomogr 1997; 21:1-7

8. Basser PJ, Jones DK. Diffusion-tensor MRI: theory, experimental design and data analysis: a technical review. NMR Biomed 2002; 15:456-67

9. Mukherjee P, Miller JH, Shimony JS, et al. Diffusion-tensor MR imaging of gray and white matter development during normal human brain maturation. AJNR Am J Neuroradiol 2002;23;1445-56

10. Cheung MM, Hui ES, Chan KC, et al. Does diffusion kurtosis imaging lead to better neural tissue characterization? A rodent brain maturation study. Neuroimage 2009;45:386-92

11. Yoshida S, Oishi K, Faria AV, et al. Diffusion tensor imaging of normal brain development. Pediatr Radiol 2013;43:15-27

12. Song SK, Sun SW, Ramsbottom MJ, et al. Dysmyelination revealed through MRI as increased radial (but unchanged axial) diffusion of water. Neuroimage 2002;17:1429-36

13. Basser PJ. Inferring microstructural features and the physiological state of tissues from diffusion-weighted images. NMR Biomed 1995;8:333-44

14. Hüppi PS, Dubois J. Diffusion tensor imaging of brain development. Semin Fetal Neonatal Med 2006;11:489-97

15. Jones DK, Knosche TR, Turner R. White matter integrity, fiber count, and other fallacies: the do's and don'ts of diffusion MRI. Neuroimage 2013;73:239-54

16. Schimrigk SK, Bellenberg B, Schluter M. Diffusion tensor imagingbased fractional anisotropy quantification in the corticospinal tract of patients with amyotrophic lateral sclerosis using a probabilistic mixture model. AJNR Am J Neuroradiol 2007;28:724-30

17. Beaulieu C. The basis of anisotropic water diffusion in the nervous system - a technical review. NMR Biomed 2002;15:435-55

18. Veraart J, Poot DH, Van Hecke W, et al. More accurate estimation of diffusion tensor parameters using diffusion kurtosis imaging. Magn Reson Med 2011;65:138-45

19. Jensen JH, Helpern JA. MRI quantification of non-Gaussian water diffusion by kurtosis analysis. NMR Biomed 2010;23:698-710

20. Lu H, Jensen JH, Ramani A, et al. Three-dimensional characterization of non-Gaussian water diffusion in humans using diffusion kurtosis imaging. NMR Biomed 2006;19:236-47

21. Tournier JD, Yeh CH, Calamante F, et al. Resolving crossing fibers using constrained spherical deconvolution: validation using diffusion-weighted imaging phantom data. Neuroimage 2008;42:617-25
22. Perrin M, Poupon C, Cointepas $\mathrm{Y}$, et al. Fiber tracking in Q-ball fields using regularized particle trajectories. Inf Process Med Imaging 2005;19:52-63

23. Jensen JH, Helpern JA, Ramani A, et al. Diffusional kurtosis imaging: the quantification of non-Gaussian water diffusion by means of magnetic resonance imaging. Magn Reson Med 2005; 53:1432-40

24. De Santis S, Gabrielli A, Palombo M, et al. Non-Gaussian diffusion imaging: a brief practical review. Magn Reson Imaging 2011; 29:1410-16

25. Tabesh A, Jensen JH, Ardekani BA, et al. Estimation of tensors and tensor-derived measures in diffusional kurtosis imaging. Magn Reson Med 2011;65:823-36

26. Lazar M, Jensen JH, Xuan L, et al. Estimation of the orientation distribution function from diffusional kurtosis imaging. Magn Reson Med 2008;60:774-81

27. Mori S, Crain BJ, Chacko VP, et al. Three-dimensional tracking of axonal projections in the brain by magnetic resonance imaging. Ann Neurol 1999;45:265-69

28. Provenzale JM, Isaacson J, Chen S. Progression of corpus callosum diffusion-tensor imaging values during a period of signal changes consistent with myelination. AJR Am J Roentgenol 2012;198: 1403-08

29. Sampaio RC and Truwit CL. Myelination of the developing human brain. In: Nelson CA, Luciana M, eds. Handbook of Developmental Cognitive Neuroscience. Cambridge, Massachusetts: MIT Press; 2001:35-44

30. Müller K, Homberg V, Lenard HG. Magnetic stimulation of motor cortex and nerve roots in children: maturation of cortico-motoneuronal projections. Electroencephalogr Clin Neurophysiol 1991;81: 63-70

31. Nezu A, Kimura S, Uehara S, et al. Magnetic stimulation of motor cortex in children: maturity of corticospinal pathway and problem of clinical application. Brain Dev 1997;19:176-80

32. Hui ES, Cheung MM, Qi L, et al. Toward better MR characterization of neural tissues using directional diffusion kurtosis analysis. $\mathrm{Neu}$ roimage 2008; $42: 122-34$

33. Gao Y, Zhang Y, Wong CS, et al. Diffusion abnormalities in temporal lobes of children with temporal lobe epilepsy: a preliminary diffusional kurtosis imaging study and comparison with diffusion tensor imaging. NMR Biomed 2012;25:1369-77

34. Helpern JA, Adisetiyo V, Falangola MF, et al. Preliminary evidence of altered gray and white matter microstructural development in the frontal lobe of adolescents with attention-deficit hyperactivity disorder: a diffusional kurtosis imaging study. J Magn Reson Imaging 2011;33:17-23 